\title{
Seleção para peso pós-desmama em um rebanho Gir. 1. Resposta direta e correlacionada em 21 anos de seleção ${ }^{1}$
}

\author{
Fabiana Batalha Knackfuss ${ }^{2}$, Alexander George Razook ${ }^{3}$, Maria Eugênia Zerlotti \\ Mercadante $^{3}$, Joslaine Noely dos Santos Gonçalves Cyrillo ${ }^{3}$, Leopoldo Andrade de \\ Figueiredo ${ }^{3}$, Humberto Tonhati 4,5
}

\author{
1 Parte da dissertação apresentada pela primeira autora ao curso de pós-graduação em Genética e Melhoramento Animal UNESP - \\ Jaboticabal - SP. \\ 2 Bolsista CAPES. \\ 3 Instituto de Zootecnia - Centro APTA de Bovinos de Corte - Estação Experimental de Zootecnia de Sertãozinho - http://www.iz.sp.gov.br/ \\ eezooser. \\ ${ }^{4}$ Depto. Melhoramento Genético Animal - FCAV - UNESP - Jaboticabal. \\ ${ }^{5}$ Bolsista do CNPq.
}

RESUMO - A seleção para peso pós-desmama tem sido aplicada em um rebanho Gir (80 vacas, seis touros) desde 1976 , e um rebanho Nelore Controle, $\mathrm{NeC}$ (60 vacas, quatro touros), tem sido mantido para avaliação da mudança ambiental. O critério de seleção dos machos foi o peso aos 378 dias (P378), obtido em prova de ganho de peso, e o das fêmeas, o peso aos 550 dias (P550) em recria a pasto. A média dos diferenciais de seleção efetivos dos pais foi, respectivamente, 19,5 e 1,8 kg para Gir e $\mathrm{NeC}$, correspondendo a 0,72 e 0,07 unidades de desvio-padrão (u.d.p.). A média dos diferenciais de seleção efetivos acumulados dos pais, na progênie de 2001, foi 83,7 e 7,2 kg para Gir e NeC, correspondendo a 3,17 e 0,29 u.d.p. O intervalo de geração médio no rebanho Gir foi 5,65 anos e no NeC 5,0 anos e o coeficiente de geração médio individual na progênie de 2001 atingiu 4,25 (Gir) e 4,65 (NeC) gerações de seleção. As estimativas de tendência genética no rebanho Gir, obtidas por quadrados mínimos, foram $\mathrm{PN}=0,16 ; \mathrm{P} 210=0,81 \mathrm{~kg} / \mathrm{ano} ; \mathrm{GPRE}=3,02 \mathrm{~g} / \mathrm{dia} / \mathrm{ano} ; \mathrm{P} 378=2,88 ; \mathrm{P} 550=2,80 \mathrm{~kg} / \mathrm{ano} ; \mathrm{G} 112=11,2$ $\mathrm{g} /$ dia/ano, $\mathrm{AM}=0,003$ e $\mathrm{AF}=0,004 \mathrm{~m} / \mathrm{ano}$. Com base no modelo misto, as estimativas para essas características foram menores (0,03 e 0,28 kg/ano; $1,15 \mathrm{~g} / \mathrm{dia} / \mathrm{ano} ; 1,45$ e 1,07 kg/ano; 3,73 g/dia/ano; 0,003 e 0,002 m/ano). As estimativas de herdabilidade realizadas para P378 e P550 foram 0,69 e 0,64, respectivamente. Os resultados indicaram que a seleção para peso pós-desmama, com base na informação de desempenho individual, promoveu respostas genéticas significativas tanto nas características sob seleção direta quanto nas respostas correlacionadas em características de crescimento da fase pré e pós-desmama no rebanho Gir.

Palavras-chave: bovinos de corte, coeficiente de geração, intensidade de seleção, mudança genética, população controle

\section{Selection for yearling weight in Gir cattle. 1. Direct and correlated response in 21 years of selection}

\begin{abstract}
Selection for yearling weight has been applied in a herd of Gir breed ( 80 cows, 6 sires) since 1976 and a Nelore Control herd ( 60 cows, 4 sires) has been used to evaluate the environmental change. Selection criteria were bull weight at 378 days (P378) in a performance test and heifer weight at 550 days (P550), under grazing conditions. The effective midparent selection differentials for P378 and P550 were 19.5 and $1.8 \mathrm{~kg}$ respectively for Gir and $\mathrm{NeC}$, corresponding to 0.72 and 0.07 standard deviation units (s.d.u). The midparent cumulative effective selection differentials for the 2001 offspring were respectively 83.7 and $7.2 \mathrm{~kg}$ for Gir and $\mathrm{NeC}$, corresponding to 3.17 and 0.29 s.d.u. The average generation intervals were 5.65 and 5.0 years respectively for the Gir and $\mathrm{NeC}$ herds. The individual generation coefficient for the 2001 offspring attained the values of 4.25 (Gir) and $4.65(\mathrm{NeC})$. Annual genetic trends estimated by least square methodology in the Gir herd were - PN: 0.16; P210: 0.81 kg/year; GPRE: 3.02 g/day/year; P378: 2.88; P550: $2.80 \mathrm{~kg} /$ year; G112: $11.2 \mathrm{~g} / \mathrm{day} / \mathrm{year}$; AM: 0.003 and AF: $0.004 \mathrm{~m} /$ year. Using the mixed model methodology, the estimates for these traits were lower, 0.03 , and $0.28 \mathrm{~kg} / \mathrm{year} ; 1.15 \mathrm{~g} / \mathrm{day} / \mathrm{year}$; 1.45 and $1.07 \mathrm{~kg} /$ year; $3.73 \mathrm{~g} /$ day/year; 0.003 and $0.002 \mathrm{~m} /$ year. The realized heritability was 0.69 and 0.64 for P378 and P550, respectively. The results indicated significant genetic response from direct selection based on individual performance information for postweaning weight as well as correlated response in pre and postweaning growth traits in a Gir herd.
\end{abstract}

Key Words: beef cattle, control population, generation coefficient, genetic change, selection intensity 


\section{Introdução}

A pressão sobre a pecuária de corte brasileira para que se torne mais eficiente tem refletido na busca por raças e sistemas de produção competitivos e sustentáveis.

Mesmo sendo composta, na grande maioria, por raças zebuínas, altamente rústicas e adaptadas ao clima tropical e responsável por aproximadamente $9 \%$ do produto interno bruto (PIB), a pecuária nacional apresenta índices incompatíveis com as demandas impostas por uma economia cada vez mais globalizada.

Neste contexto, observa-se ainda carência de programas de seleção com bovinos de corte, estabelecidos para fornecer evidências objetivas sobre respostas diretas e correlacionadas para características de interesse econômico.

O Instituto de Zootecnia mantém, na Estação Experimental de Zootecnia de Sertãozinho (SP), um programa de seleção para características de crescimento em três raças zebuínas: Nelore, Gir e Guzerá. Os rebanhos destas três raças começaram a ser formados em 1933 e, até 1976, a seleção foi realizada com base, principalmente, em características raciais. Como não havia preocupação com a avaliação do progresso genético, em 1976 foi elaborado um projeto de seleção para estas raças, cujo critério de seleção foi o peso aos 18 meses, pelo qual avaliou-se a mudança genética com o auxílio de uma população controle, nãoselecionada.

Desde a implantação do projeto, diversos estudos sobre respostas diretas no peso pós-desmama (Packer et al., 1986, Razook et al., 1998) e as correlacionadas a medidas corporais (Cyrillo et al., 2000) e reprodutivas (Mercadante et al., 2003) foram desenvolvidos para as raças Nelore e Guzerá. Entretanto, até o momento, nenhum trabalho de avaliação das mudanças genéticas ocorridas no rebanho Gir foi realizado.

Os objetivos neste trabalho foram avaliar as intensidades de seleção praticadas e as respostas diretas e correlacionadas em progênies nascidas no período de 1981 a 2001 em um rebanho da raça Gir utilizando-se métodos de quadrados mínimos e de modelo misto.

\section{Material e Métodos}

Os dados utilizados foram provenientes de um rebanho Gir e de um rebanho Nelore controle $(\mathrm{NeC})$, que fazem parte do programa de seleção das raças zebuínas, desenvolvido pela Estação Experimental de Zootecnia de Sertãozinho (EEZS), localizada em Sertãozinho, SP, unidade da Secretaria da Agricultura do Estado de São Paulo. Localizada aonorte do estado de São Paulo (2 $1^{\circ} 10^{\prime}$ ' latitude norte e $47^{\circ} 57^{\prime}$ longitude oeste), a estação apresenta clima tropical úmido, com temperatura e precipitação média anual de $24^{\circ} \mathrm{C}$ e $1.312 \mathrm{~mm}$. As pastagens são constituídas por variedades dos capins colonião (Pannicum maximum) e braquiária (Brachiaria brizanta).

O processo de reestruturação dos rebanhos Gir e Nelore e do sistema de manejo, tendo em vista a primeira estação de monta, foi iniciado em 1976. Na progênie resultante desta estação de monta, nascida em 1981, foi realizada a primeira seleção (Razook et al., 1998). O primeiro procedimento adotado foi a introdução de material genético de outras linhagens, com o intuito de promover maior variabilidade genética nos rebanhos, evitando altos níveis de endogamia no decorrer dos anos de seleção.

A escolha dos touros fundadores, nascidos de 1977 a 1980, tanto no rebanho Gir como no NeC, baseou-se no critério de seleção vigente na época (peso aos 550 dias). Em 1980, o rebanho Nelore, até então único, foi dividido em três rebanhos, sendo um deles o $\mathrm{NeC}$, com 60 vacas e quatro touros, estabelecido com a finalidade de monitorar as mudanças ambientais. No rebanho Gir, o número de matrizes foi igual a 80 .

Anualmente, foram selecionados três tourinhos Gir (equivalentes a $10 \%$ do grupo contemporâneo), com base no máximo diferencial de seleção do peso aos 378 dias (P378), obtido em prova de ganho de peso (PGP), e de 15 a 20 vacas (equivalente a 50-60\% do grupo contemporâneo), com base no peso aos 550 dias, obtido em recria a pasto. No rebanho $\mathrm{NeC}$, os reprodutores foram selecionados com base em diferenciais de seleção próximos de zero para estes mesmos critérios. A estação de monta teve duração de 90 dias, da primeira quinzena de novembro à primeira quinzena de fevereiro. Os rebanhos, que na maior parte do ano receberam o mesmo manejo, nesta época foram separados em lotes de acasalamento com um único touro e mantidos em piquetes distintos. Os nascimentos ocorreram, em média, da segunda quinzena de agosto até meados de novembro. Após o desmame, feito aos 7 meses de idade (abril-maio), os machos foram encaminhados à PGP, onde permaneceram sob regime de confinamento por um período de 168 dias, quando foram selecionados com base no seu peso final (P378). As fêmeas foram mantidas em pastagem até completarem 18 meses (550 dias), quando foram selecionadas.

Para as análises utilizando-se a metodologia dos quadrados mínimos, foram considerados registros de 2.090 bovinos pertencentes aos rebanhos Gir (1125) e NeC (965), nascidos de 1981 a 2001, filhos de 116 touros (67 Gir e 49 $\mathrm{NeC}$ ) e 551 matrizes (321 Gir e $230 \mathrm{NeC}$ ). 
Para as análises utilizando-se a metodologia dos modelos mistos, foram considerados os registros dos animais nascidos de 1978 a 2001 no rebanho Gir e de 1981 a 2001 no NeC. Também foram utilizados arquivos depedigree de animais nascidos de 1934 a 2001 para o rebanho Gir e de 1958 a 2001, para o $\mathrm{NeC}$, voltando até a 10 a geração dos animais com registros. Para o rebanho Gir, o arquivo depedigree incluiu 1.740 registros, sendo 93 animais-base (sem informação de pai e mãe), enquanto, para o $\mathrm{NeC}$, incluiu 1.980 registros, dos quais 385 eram animais-base.

As características avaliadas neste estudo foram: peso ao nascer $(\mathrm{PN})$, peso à desmama corrigido para 210 dias (P210), ganho diário na fase pré-desmama (GPRE), peso de machos ao final da prova de ganho de peso (P378), ganho diário obtido na prova de ganho de peso em confinamento (G112), peso de fêmeas corrigido para 550 dias (P550), altura aos 378 dias em machos (AM) e altura aos 550 dias em fêmeas (AF).

Os diferenciais de seleção esperados foram calculados como o desvio do desempenho individual no P378 (machos) e no P550 (fêmeas) em relação à média dos contemporâneos e os diferenciais de seleção efetivos, como a média ponderada dos diferenciais esperados pelo número de filhos efetivamente avaliados no critério de seleção (Dickerson et al., 1954).

As respectivas intensidades de seleção foram obtidas pela divisão dos diferenciais de seleção esperados pelo desvio-padrão da característica de seleção direta, dentro dos grupos contemporâneos.

Os diferenciais de seleção efetivos acumulados de touros (DSAT) e vacas (DSAV), bem como as respectivas intensidades de seleção efetivas acumuladas (iEAT e iEAV), foram obtidos pelo método descrito por Newman et al. (1973) da seguinte forma: DAS = DSE + DSEAMP, em que: $\mathrm{DAS}=$ diferencial de seleção acumulado no indivíduo; DSE = diferencial de seleção esperado; DSEAMP = diferencial de seleção efetivo acumulado médio dos pais, cuja ponderação foi feita sobre todos indivíduos do grupo contemporâneo dos pais. Os animais fundadores foram designados como tendo DSA igual a zero e o DSEAMP foi obtido pela média aritmética dos diferenciais de seleção efetivos acumulados de touros (DSEAT) e vacas (DSEAV), respectivamente.

O intervalo de geração (IG) foi obtido pela ponderação da idade dos touros e das vacas pelo número de suas progênies em determinado ano da seguinte forma:

$$
\mathrm{IG}=\left(\left(\sum \mathrm{t}_{\mathrm{i}}(\mathrm{IT})_{\mathrm{i}}+\sum \mathrm{v}_{\mathrm{i}}(\mathrm{IV})_{\mathrm{i}} / 2 \mathrm{~N}\right)\right.
$$

em que: $(I T)_{i}$ e $(I V)_{i}=$ classes de idades de touros e vacas ao nascimento de determinada progênie; $\mathrm{t}_{\mathrm{i}} \mathrm{ev}_{\mathrm{i}}=$ número de progênies por classe de idade de touro e vaca; $\mathrm{N}$ = número total de progênies em determinado ano.

Em espécies que apresentam sobreposição de gerações, como os bovinos, indivíduos contemporâneos podemdiferir quanto à geração. $\mathrm{O}$ valor representativo da geração a que o indivíduo pertence, também denominado coeficiente de geração, corresponde ao número de segregações mendelianas a partir dos animais fundadores. Os coeficientes de geração foram calculados de acordo com a fórmula sugerida por Brinks et al. (1965): CGI $=((\mathrm{CGT}+\mathrm{CGV}) / 2)+1$, em que: $\mathrm{CGI}=$ coeficiente de geração do indivíduo; $\mathrm{CGT}=$ coeficiente de geração do touro; $\mathrm{CGV}=$ coeficiente de geração da vaca. Os animais fundadores têm coeficiente de geração individual (CGI) igual a zero.

Até 1993, o parentesco entre touros e vacas foi estimado pelo exame de fichas de genealogia. Posteriormente, passou a ser feito pelo programa computacional SIREGE (Sis tema para Relacionamento Genético Animal), desenvolvido por Polastre et al. (1992).

As análises estatísticas foram conduzidas sob duas metodologias distintas: a dos quadrados mínimos (QM), utilizando-se os programas computacionais SAS (1999) e HARVEY (1990) e a metodologia de modelos mistos (MM) sob modelo animal, utilizando-se o programa MTDFREML (Boldman et al., 1995).

Uma série de análises preliminares foi realizada até a definição de modelos que melhor explicassem a variação das referidas características na população. Assim, inicialmente, foram testados os efeitos fixos de ano, sexo, mês de nascimento, rebanho e idade da vaca, além de todas as interações duplas possíveis entre os efeitos principais, sendo mantidos no modelo final aqueles efeitos com significância $(\mathrm{P}<0,05)$.

O modelo definido foi o seguinte:

$\mathrm{Y}_{\mathrm{ijklmn}}=\mu+\mathrm{a}_{\mathrm{i}}+\mathrm{c}_{\mathrm{j}}+\mathrm{f}_{\mathrm{k}}+\mathrm{r}_{\mathrm{l}}+\mathrm{v}_{\mathrm{m}}+(\mathrm{ar})_{\mathrm{il}}+\mathrm{e}_{\mathrm{ijklmn}}$ (1) em que: $Y_{i j k l m n}=$ valor observado; $\mu=$ constante comum a todos os indivíduos para cada característica; $\mathrm{a}_{\mathrm{i}}=$ efeito do ano de nascimento do animal $(\mathrm{i}=1981, \ldots, 2001) ; \mathrm{c}_{\mathrm{j}}=$ efeito do mês de nascimento $(j=8, \ldots, 10$ e $j \geq 11) ; f_{k}=$ efeito de sexo em características da fase pré-desmama $(\mathrm{k}=1,2) ; \mathrm{r}_{1}=$ efeito do rebanho $(1=1,2) ; \mathrm{v}_{\mathrm{m}}=$ efeito da idade da vaca em anos $(\mathrm{m}=3, \ldots, 10$ e $\mathrm{m} \geq 11) ;(\text { ar })_{\mathrm{il}}=$ interação do ano de nascimento e rebanho; $\mathrm{e}_{\mathrm{ijk} \operatorname{lnm}}=$ erro aleatório associado a cada observação do indivíduo.

A metodologia QM também foi utilizada para obtenção dos coeficientes de regressão e das médias de cada característica em função do ano de nascimento, necessários para a obtenção das tendências fenotípicas e genéticas em cada rebanho. Pelo desdobramento do efeito do ano de nascimento, 
para cada rebanho, em polinômios ortogonais conforme procedimento utilizado no programa HARVEY (1990), foi possível obter as equações que descrevem essas regressões .

O modelo utilizado foi o seguinte:

$$
\mathrm{Y}_{\mathrm{ijklmn}}=\mu+\mathrm{c}_{\mathrm{j}}+\mathrm{f}_{\mathrm{k}}+\mathrm{r}_{\mathrm{l}}+\mathrm{v}_{\mathrm{m}}+\mathrm{a}_{\mathrm{il}}+\mathrm{e}_{\mathrm{ijklmn}}
$$

em que: $m, c_{j}, f_{k}, r_{1}, v_{m}, e_{i j k l m n}$, como descritos anteriormente; $\mathrm{a}_{\mathrm{il}}=$ efeito de ano $\mathrm{i}$ de nascimento do animal dentro de rebanho 1 (subclasse $i=1981, \ldots, 2001$ ) para $1(1,2)$. Para que isso fosse possível, foi criada uma codificação adicional de ano dentro de rebanho, agrupando-se o código de rebanho ao de ano.

Pelo método QM, a resposta genética anual no rebanho Gir foi estimada como o desvio das médias anuais ajustadas desse rebanho em relação às médias do $\mathrm{NeC}$, ambas obtidas conforme o modelo 1. A tendência genética foi estimada como a diferença entre os coeficientes de regressão linear das médias anuais de cada característica, em função do ano de nascimento do rebanho Gir em relação ao do rebanho $\mathrm{NeC}$. Os coeficientes de regressão para os dois rebanhos foram obtidos sob o modelo 2. Neste método, pressupõe-se a não-ocorrência de mudança genética no rebanho $\mathrm{NeC} \mathrm{e}$ que a mudança observada neste rebanho equivale a uma estimativa de mudança ambiental, igual à estimativa de mudança ambiental no rebanho Gir, assumindo-se que não há interação genótipo-ambiente, uma vez que os rebanhos Gir e NeC são geneticamente diferentes.

Pela metodologia MM, a estimativa de resposta genética anual foi obtida pela média dos valores genéticos diretos para cada ano de nascimento, obtidos por meio de análises univariadas para todas as características da fase pré-desmama no rebanho Gir. Para essas características, o modelo misto incluiu os efeitos fixos de mês de nascimento, grupo contemporâneo e idade da mãe, em anos, além dos efeitos aleatórios genético direto do animal, genético materno e de ambiente permanente materno. Análises preliminares testando cinco diferentes modelos (pelo teste de razão de verossimilhança) indicaram ser este o melhor modelo para estas características.

Em notação matricial, o modelo utilizado pode ser descrito como:

$$
\mathrm{Y}=\mathrm{X} \beta+\mathrm{Z}_{1} \mathrm{a}+\mathrm{Z}_{2} \mathrm{~m}+\mathrm{Z}_{3} \mathrm{p}+\mathrm{e}
$$

em que: $Y=$ vetor de observações; $\beta=$ vetor de efeitos fixos; $\mathrm{a}=$ vetor de efeito aleatório genético aditivo direto; $\mathrm{m}=$ vetor de efeito aleatório genético aditivo materno; $\mathrm{p}=$ vetor de efeitos de ambiente permanente; e = vetor de efeitos residuais; $\mathrm{X}, \mathrm{Z}_{1}, \mathrm{Z}_{2}$ e $\mathrm{Z}_{3}$ = matrizes de incidência associadas a $\beta$, a, m e p, respectivamente.

Como proposto por Mercadante et al. (2003), os valores genéticos para P378 e P550 foram obtidos por análise bicaráter entre as características (P378-P550), assim como para ganho de peso (G112-GPRE) e alturas em machos e fêmeas (AM-AF). A resposta genética anual no rebanho NeC foi obtida somente para P378 e P550, cujos valores genéticos foram preditos por meio de análises bivariadas. Tanto no rebanho Gir quanto no rebanho NeC, o modelo misto incluiu os mesmos efeitos fixos descritos para as características da fase pré-desmama, além do efeito aleatório genético direto. As tendências genéticas foram obtidas pela regressão das médias dos valores genéticos diretos, em função do ano de nascimento do animal.

As estimativas de herdabilidade realizadas para P378 e P550 foram obtidas pela regressão da resposta acumulada, pelos desvios em relação ao rebanho $\mathrm{NeC}$ (conforme modelo 1), em função do diferencial de seleção efetivo acumulado médio dos pais. Para este cálculo, corrigiu-se o diferencial de seleção acumulado no rebanho Gir, subtraindo-se aquele encontrado no rebanho $\mathrm{NeC}$.

\section{Resultados e Discussão}

Na Tabela 1 são apresentados os resultados da seleção aplicada e o intervalo de geração. Os valores médios dos diferenciais de seleção efetivos (DSE) para P378 e P550 nos rebanhos Gir e $\mathrm{NeC}$ foram, respectivamente, 19,5 e 1,8 kg, correspondentes a 0,72 e 0,07 unidades de desvio-padrão (u.d.p). Esses valores, que expressam a superioridade dos touros e das fêmeas selecionados nessas características, em relação aos contemporâneos no momento da seleção, foram inferiores aos encontrados por Baker et al. (1991), que corresponderam a 1,04 e 1,02 u.d.p, em rebanhos Angus selecionados para peso ao ano e aos 18 meses, respectivamente, e a 0,86 u.d.p, em rebanhos Hereford selecionados para peso ao ano. Koch et al. (1994) reportaram valor igual a 1,0 u.d.p em bovinos da raça Hereford selecionados para peso pós-desmama.

No rebanho Gir, a contribuição de touros correspondeu a $87 \%$ sobre o diferencial de seleção efetivo total, comprovando a grande contribuição de touros em um programa de seleção como este. Como esperado, isso não foi verificado no rebanho $\mathrm{NeC}$, onde houve, inclusive, dificuldade de reposição de fêmeas com diferencial nulo. Observou-se, dessa forma, ocorrência de seleção não-intencional no rebanho $\mathrm{NeC}$, o que pode ter comprometido a estimativa de resposta à seleção por QM.

É conveniente destacar que tanto o diferencial de seleção quanto a intensidade de seleção são dependentes não só do número de animais selecionados, mas também da variação no grupo contemporâneo. De qualquer forma, a 
Tabela 1 - Média dos diferenciais (DSE), das intensidades (IE) de seleção efetivas, dos diferenciais (DSEA) e das intensidades de seleção acumuladas (IEA), do intervalo de gerações (IG) dos touros $(T)$, das vacas $(V)$ e da média dos pais (MP) por rebanho

Table 1 - Average effective selection differentials (DSE), intensities (IE), cumulative effective selection differentials (DSEA) and intensities (IEA) and generation interval (IG) for sire (T), dam (V) and midparent (MP) by herd

\begin{tabular}{|c|c|c|c|c|c|c|}
\hline \multirow[t]{2}{*}{ Item } & \multicolumn{3}{|c|}{ GIR } & \multicolumn{3}{|c|}{$\mathrm{NeC}^{1}$} \\
\hline & $\mathrm{T}$ & $\mathrm{V}$ & MP & $\mathrm{T}$ & V & MP \\
\hline DSE $(\mathrm{kg})$ & 34,0 & 5,1 & 19,5 & $-0,1$ & 3,7 & 1,8 \\
\hline IE (u.d.p $)^{2}$ & 1,20 & 0,24 & 0,72 & $-0,01$ & 0,15 & 0,07 \\
\hline DSEA- $2001^{3}(\mathrm{~kg})$ & 100,4 & 67,1 & 83,7 & 6,9 & 7,6 & 7,2 \\
\hline IEA-2001 (u.d.p) & 3,85 & 2,50 & 3,17 & 0,28 & 0,31 & 0,29 \\
\hline IG (anos) & 3,95 & 7,36 & 5,65 & 3,63 & 6,35 & 5,00 \\
\hline
\end{tabular}

${ }^{1} \mathrm{NeC}$ : Nelore Controle; ${ }^{2}$ u.d.p.:unidades de desvio-padrão; ${ }^{3} 2001:$ representa valores acumulados para a progênie de 2001 .

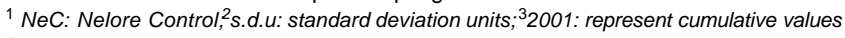
for 2001 progeny.

pressão de seleção foi efetiva no rebanho Gir, o que provocou mudança nas características selecionadas (P378 e P550).

$\mathrm{O}$ valor encontrado neste estudo para o diferencial de seleção efetivo acumulado médio dos pais para o rebanho Gir, está abaixo dos encontrados por Newman et al. (1973), em bovinos da raça Shorthorn, e por Razook et al. (2002), em bovinos das raças Nelore e Guzerá. Isso talvez tenha ocorrido pelo fato de o rebanho Gir ser menor que estes rebanhos e apresentar menor taxa de fertilidade.

$\mathrm{Na}$ Tabela 2 são apresentados os resultados dos coeficientes de geração individual e endogamia. Pelos valores de endogamia das progênies de 1981 e 2001 nos dois rebanhos, foi possível observar que, mesmo tratando-se de rebanhos fechados, os valores se mantiveram moderados, provavelmente em razão do processo de formação dos lotes de monta, nos quais se restringiu o parentesco próximo nos acasalamentos.
O maior valor $(5,65)$ para o rebanho Gir no intervalo de gerações deveu-se principalmente à maior permanência de fêmeas fundadoras em alguns anos neste rebanho.

O coeficiente de geração médio na progênie de 2001 foi de 4,25 no rebanho Gir e de 4,65 gerações no NeC, podendo-se constatar que, face ao menor intervalo de gerações no rebanho $\mathrm{NeC}$, houve também sobreposição mais rápida de gerações, ou seja, como os coeficientes da progênie avançam sempre uma geração daquele da média dos pais, quanto maior a média de idade dos pais, menor o coeficiente de geração individual da progênie.

As médias ajustadas das características avaliadas por ano de nascimento e rebanho são representadas na Figura 1. Observou-se grande variabilidade entre os anos nas médias dos dois rebanhos, sugerindo efeitos ambientais não identificados intrínsecos a cada ano.

Além disso, os dois rebanhos apresentaram comportamentos distintos em determinados anos, o que pode estar relacionado não só à qualidade das pastagens durante a estação de monta, quando as vacas não permaneceram juntas, mas indicando também que os dois rebanhos reagiram de forma diferente aos estímulos ambientais. Deve-se ressaltar que o ambiente fornecido aos animais dos dois rebanhos não foram rigorosamente os mesmos, visto que não permaneceram juntos no mesmo piquete, e, portanto pode haver efeitos ambientais advindos da qualidade das pastagens fornecidas no verão, principalmente para o grupo contemporâneo de novilhas até os 18 meses.

As mudanças genéticas nas várias características das fases pré e pós-desmama, estimadas como os desvios do rebanho Gir em relação ao $\mathrm{NeC}$ pelo método QM, são ilustradas nas Figuras 2, 4, 6, 8 e 10. Da mesma forma, as médias dos valores genéticos diretos por ano de nascimento, estimados por MM, são apresentadas nas Figuras 3, 5, 7, 9 e 11 para as mesmas características.

Tabela 2 - Coeficiente de geração (CG) e endogamia na progênie de 2001 por rebanho

Table 2 - Generation coefficient (CG) and inbreeding in 2001 progeny by herd

\begin{tabular}{|c|c|c|c|c|c|c|}
\hline & \multicolumn{3}{|c|}{ Gir } & \multicolumn{3}{|c|}{$\mathrm{NeC}^{1}$} \\
\hline & $\begin{array}{l}\text { Mínimo } \\
\text { Minimum }\end{array}$ & $\begin{array}{l}\text { Máximo } \\
\text { Maximum }\end{array}$ & $\begin{array}{l}\text { Média } \\
\text { Average }\end{array}$ & $\begin{array}{l}\text { Mínimo } \\
\text { Minimum }\end{array}$ & $\begin{array}{l}\text { Máximo } \\
\text { Maximum }\end{array}$ & $\begin{array}{l}\text { Média } \\
\text { Average }\end{array}$ \\
\hline $\begin{array}{l}\text { CG-2001(Gerações) } \\
\text { CG-2001(Generations) } \\
\text { Endogamia }(\%)\end{array}$ & 3,25 & 4,95 & 4,25 & 3,83 & 5,32 & 4,65 \\
\hline $\begin{array}{l}\text { Inbreeding }(\%) \\
\text { Progênie } 1981 \\
1981 \text { progeny }\end{array}$ & 0,5 & 25,1 & 4,2 & 0,0 & 12,1 & 3,8 \\
\hline $\begin{array}{l}\text { Progênie } 2001 \\
2001 \text { progeny }\end{array}$ & 3,3 & 7,1 & 5,2 & 2,8 & 7,4 & 5,3 \\
\hline
\end{tabular}

${ }^{1} \mathrm{NeC}$ : Nelore Controle (NeC: Nelore Control). 
PN
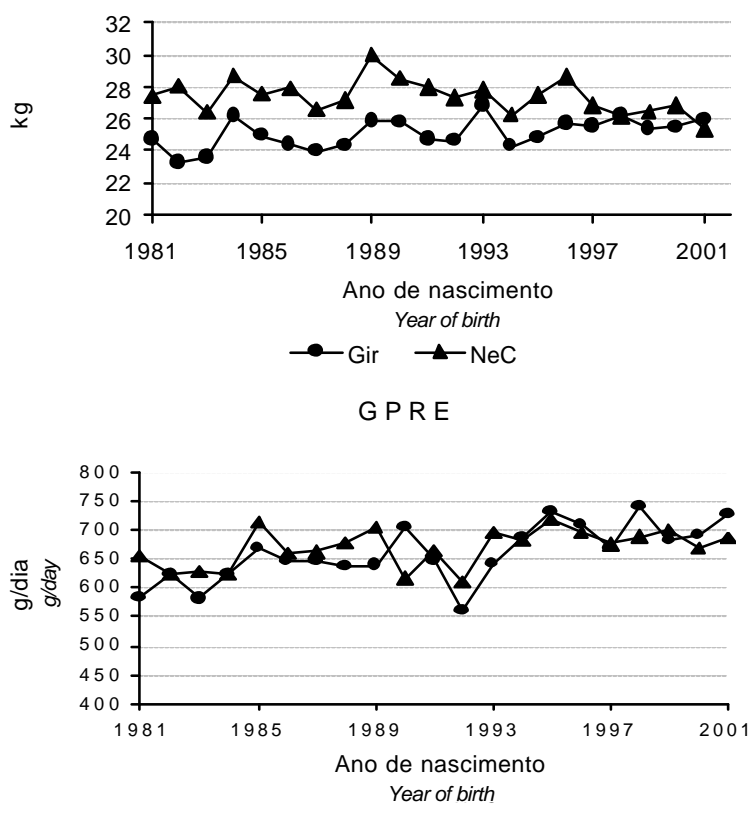

G 112

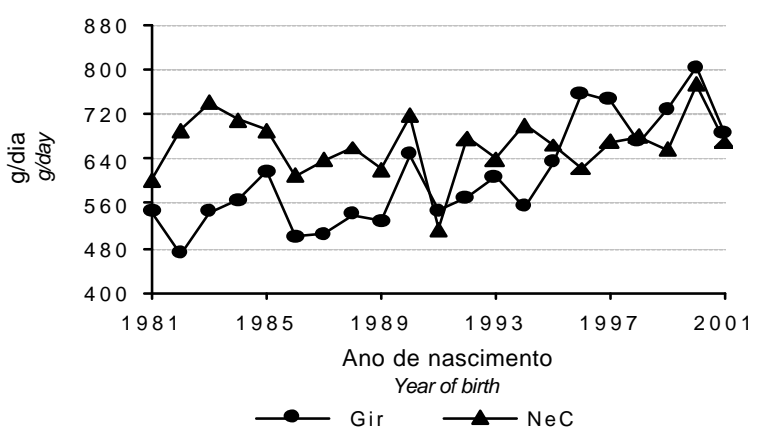

AM

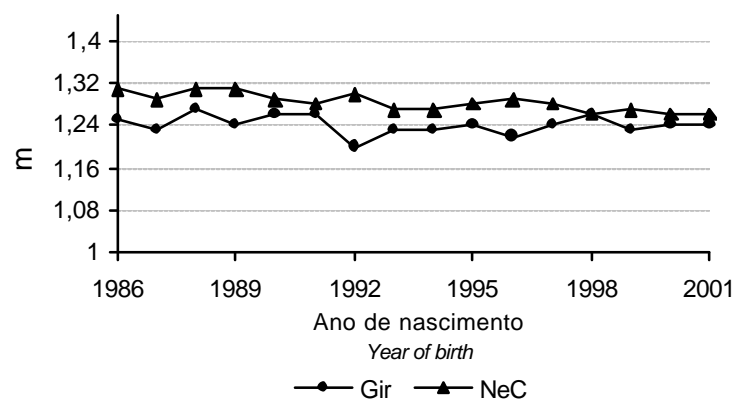

P210

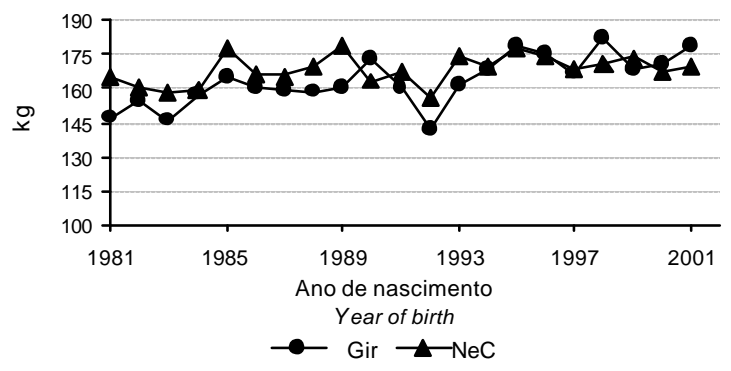

P 378

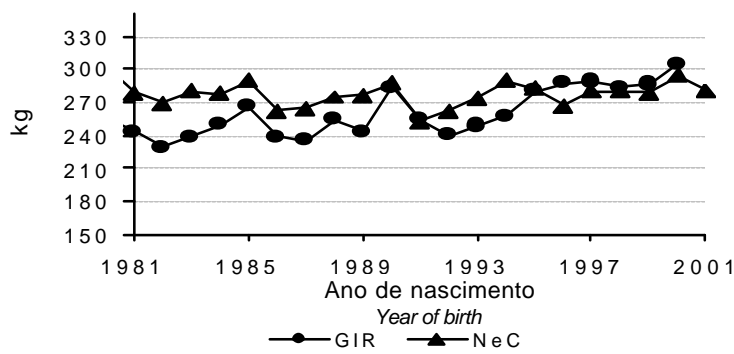

P550
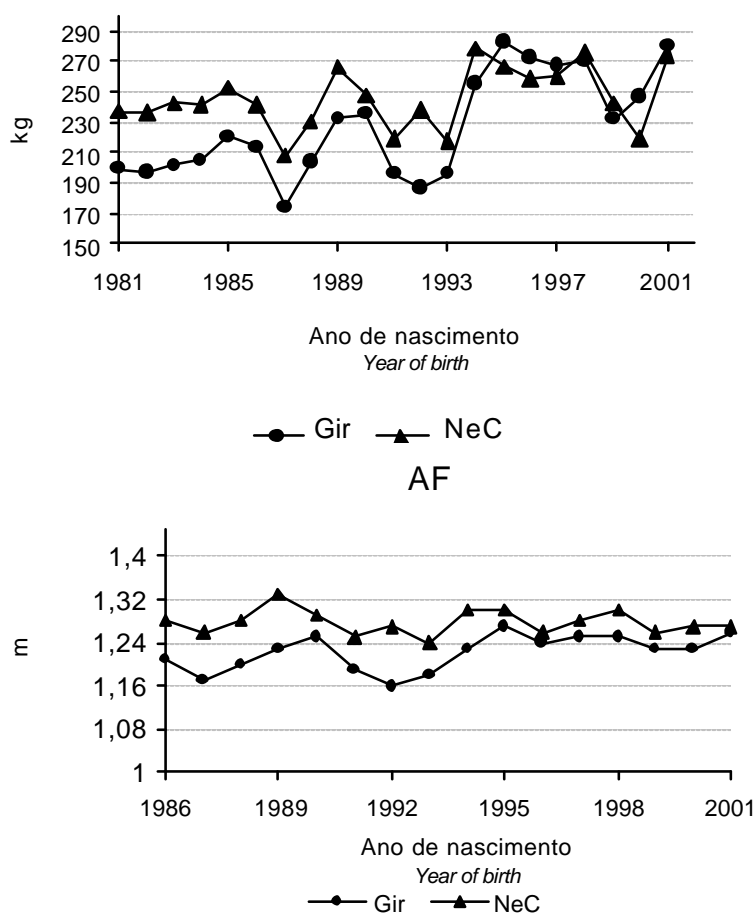

Figura 1 - Médias ajustadas do peso ao nascer (PN), do peso à desmama corrigido para 210 dias (P210), do ganho diário na fase prédesmama (GPRE), do ganho diário obtido na prova de ganho de peso (G112), do peso de machos ao final da PGP (P378), do peso de fêmeas corrigido para 550 dias (P550), da altura aos 378 dias, em machos (AM), e da altura aos 550 dias, em fêmeas $(\mathrm{AF})$, por ano de nascimento e por rebanho.

Figure 1- Least squares means for birth weight (PN); weaning weight adjusted to 210 days (P210); daily weight gain in preweaning phase (GPRE); males performance test weight at 378 days (P378); daily weight gain during the performance test (G112); heifer's weight at 550 days (P550); bulls height at 378 days (AM) and heifer height at 550 days (AF) by year of birth and herd. 


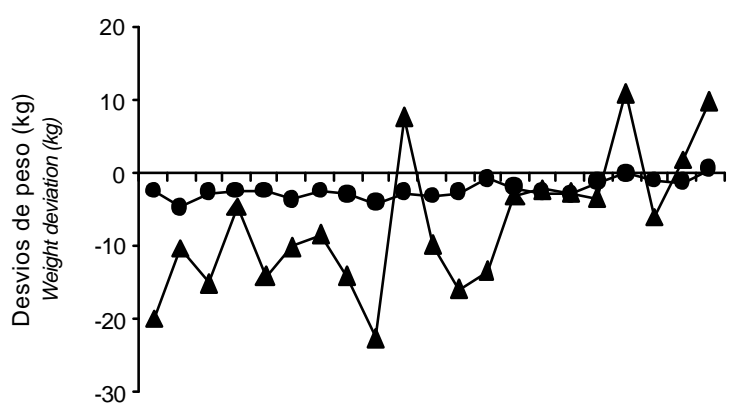

19811983198519871989199119931995199719992001

Ano de nascimento

Year of birth

Figura 2 - Desvios do peso ao nascer $(\bullet P N)$ e do peso à desmama corrigido para 210 dias (AP210) do rebanho Gir, em relação à média ajustada do $\mathrm{NeC}$.

Figure 2 - Deviation of Gir birth weight $P$ PN) and weaning weight adjusted to 210 days ( $\mathbf{A}$ 210) from $\mathrm{NeC}$ adjusted means.

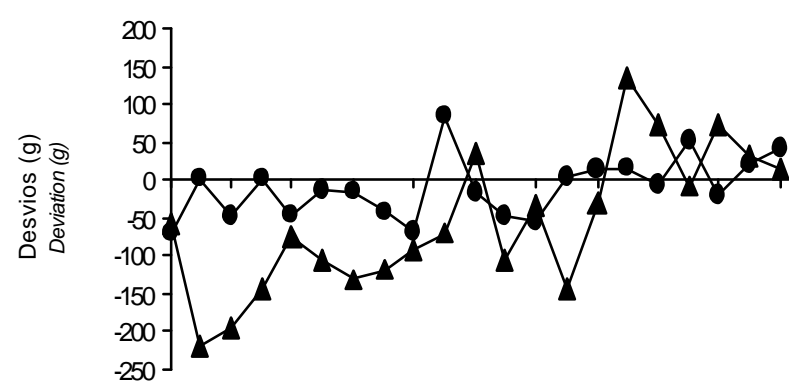

19811983198519871989199119931995199719992001

Ano de nascimento

Year of birth

Figura 4 - Desvios do ganho pré-desmama (•GPRE) e na prova de ganho de peso ( $\Delta$ G112) do rebanho Gir, em relação à média ajustada do $\mathrm{NeC}$.

Figure 4 - Deviation of Gir Daily weight gain in preweaning $(\bullet$ GPRE) and postweaning (\G112) from $\mathrm{NeC}$ adjusted means.

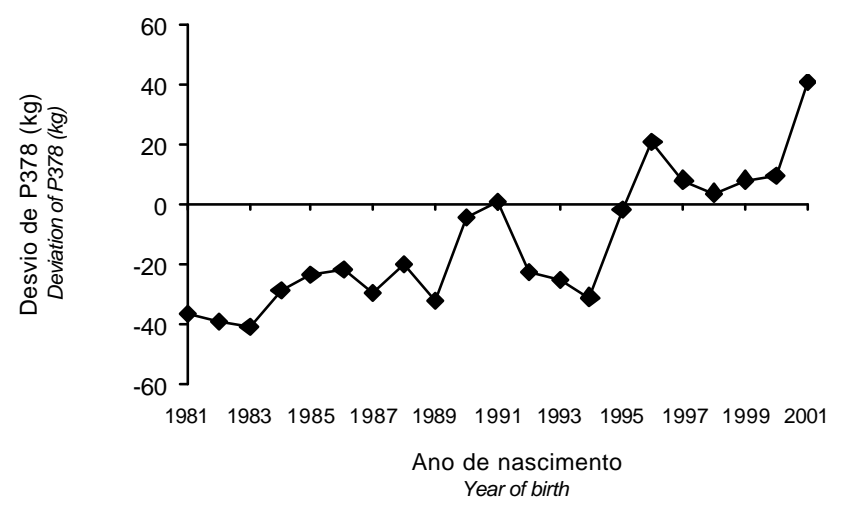

Figura 6 - Desvios do peso de machos ao final da prova de ganho de peso (P378) do rebanho Gir, em relação à média ajustada do $\mathrm{NeC}$.

Figure 6 - Deviations of the male performance testing weight at 378 days (P378) of the Gir from NeC adjusted means.

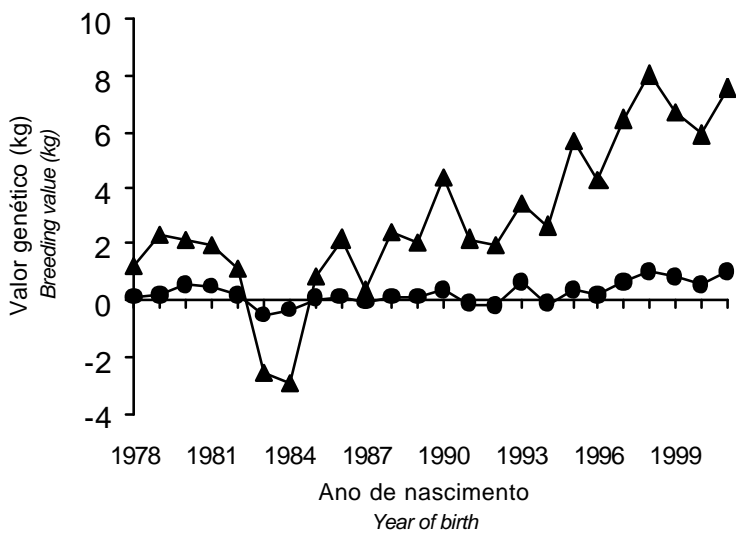

Figura 3 - Médias anuais dos valores genéticos do peso ao nascer ( $\bullet P N)$ e do peso à desmama corrigido para 210 dias ( $\mathbf{A}$ 210) do rebanho Gir.

Figure 3 - Annual mean breeding values for birth weight $P P N)$ and weaning weight corrected to 210 days ( $\mathbf{\Delta}$ P210) of the Gir herd.

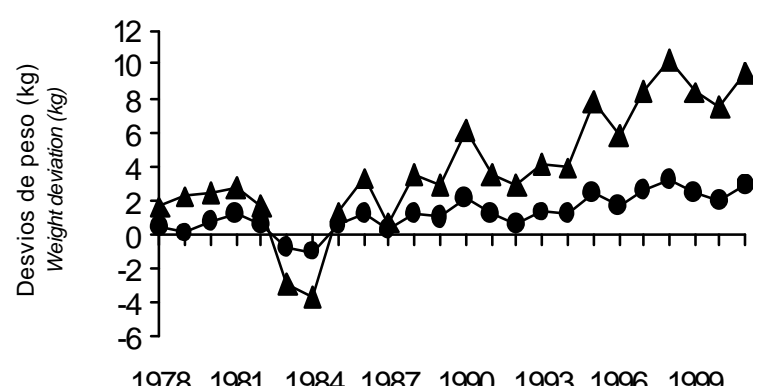

Ano de nascimento

Year of birth

Figura 5 - Médias anuais dos valores genéticos dos ganhos na fase pré-desmama ( $\bullet$ GPRE) e na prova de ganho de peso ( $\Delta$ G112) do rebanho Gir.

Figure 5 - Annual mean breeding values of daily weight gains in preweaning $(\bullet \mathrm{GPRE})$ and postweaning ( $\Delta$ G112) of the Gir herd.

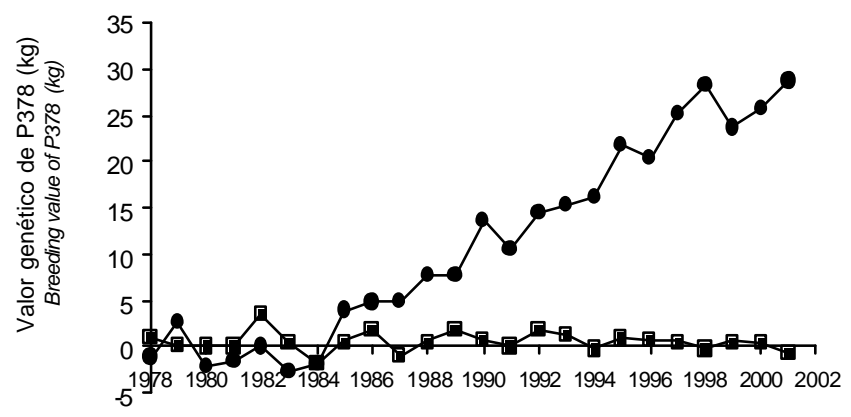

Ano de nascimento

Year of birth

Figura 7 - Médias anuais dos valores genéticos do peso de machos ao final da prova de ganho de peso (P378) por rebanho (•Gir, $\left.\square^{N e C}\right)$.

Figure 7 - Annual mean breeding values of the male's performance testing weight (P378) by herd $\left(\bullet \mathrm{Gir}, \mathbf{\otimes}_{\mathrm{NeC}}\right)$.

(C) 2006 Sociedade Brasileira de Zootecnia 


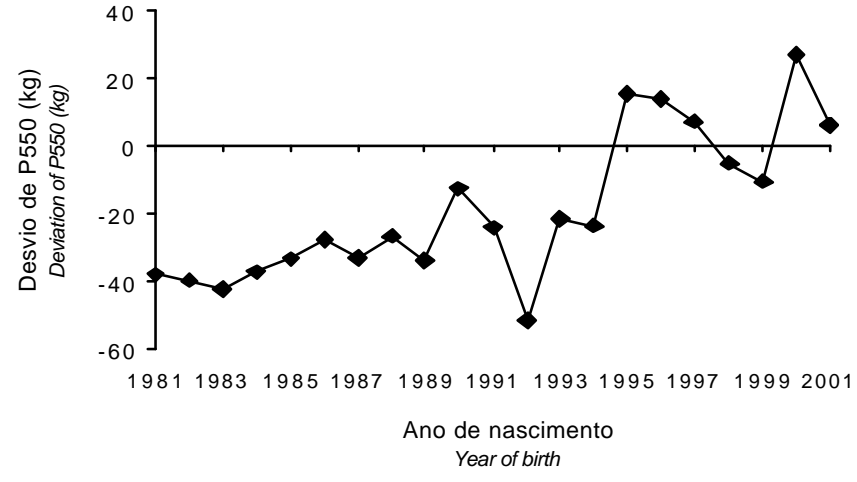

Figura 8 - Desvios do peso aos 550 dias de idade (P550) do rebanho Gir, em relação à média ajustada do $(\mathrm{NeC})$.

Figure 8 - Deviation of the Gir heifer's weight at 550 days from $\mathrm{NeC}$ adjusted means.

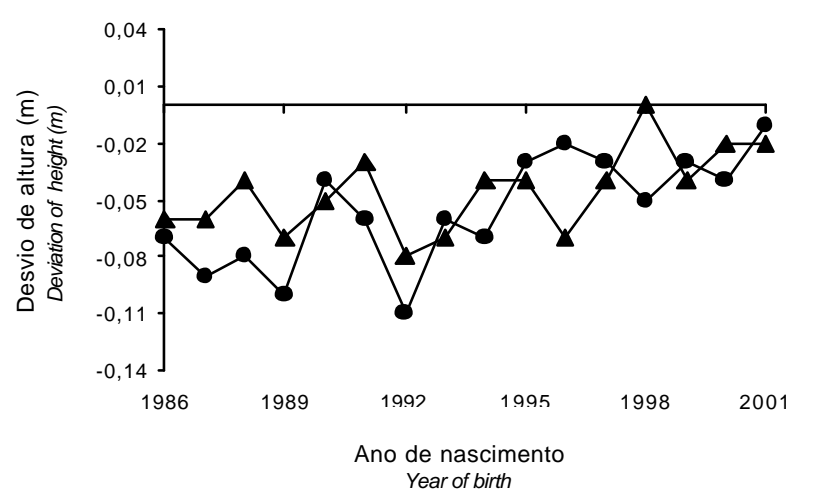

Figura 10 - Desvios da altura em machos aos 378 dias ( $\mathbf{\Delta}$ AM) e em fêmeas aos 550 dias $(\bullet A F)$ do rebanho Gir, em relação à média ajustada do $\mathrm{NeC}$.

Figure 10 - Deviation of the Gir bull height at 378 days $(\mathbf{\Delta} A M)$ and heifer's height at 550 days $(\bullet \mathrm{AF})$ from $\mathrm{NeC}$ adjusted means.

Os desvios do rebanho Gir em relação ao $\mathrm{NeC}$ apresentaram grande variação de ano para ano, evidenciando que os efeitos genéticos e ambientais podem estar parcialmente confundidos. Convém lembrar, no entanto, as limitações dos desvios do rebanho Gir em relação ao $\mathrm{NeC}$ como avaliação da mudança genética, não só pela seleção nãointencional e direcional no rebanho $\mathrm{NeC}$, como também na sua função de indicador de variação ambiental, pelo fato de os dois rebanhos pertencerem a raças distintas, embora tenham sido submetidos ao processo de seleção simultaneamente. Conforme destacado por Mercadante et al. (2003), a incorporação das relações de parentesco entre todos animais contornaria os problemas que contribuem para a variância da resposta à seleção - não só aqueles relacionados a fatores ambientais, não identificáveis, mas também a variância atribuída à deriva genética. De fato, observaram-

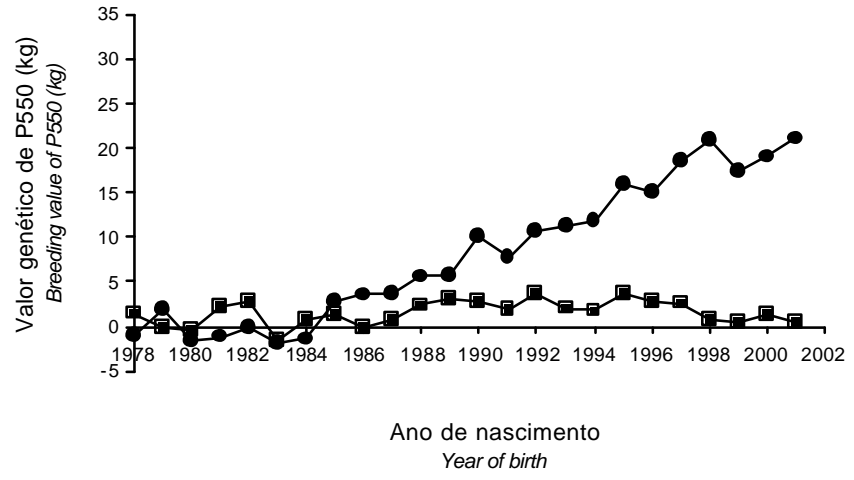

Figura 9 - Médias anuais dos valores genéticos do peso aos 550 dias de idade (P550) dos rebanhos (•Gir, $\square \mathrm{NeC}$ ).

Figure 9- Mean annual breeding values of the heifer's weight at 550 days (P550) by herd ( $\bullet$ Gir, $\mathbf{N e C}$ ).

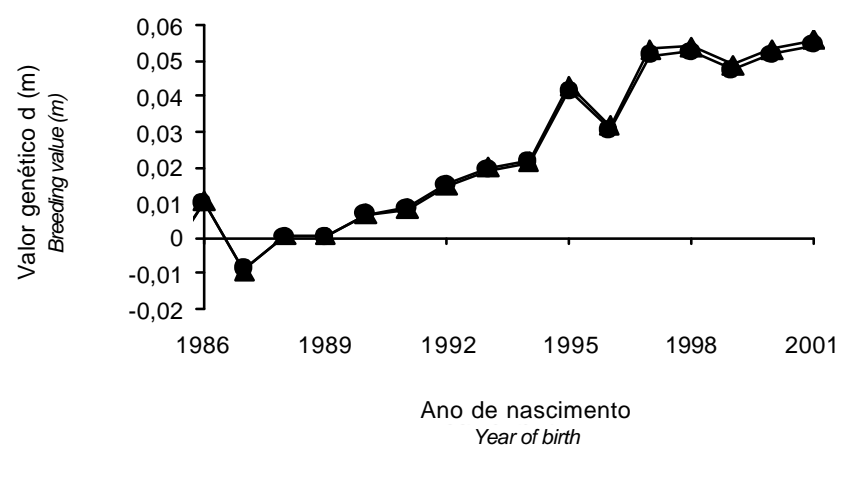

Figura 11 - Médias anuais dos valores genéticos da altura em machos $(\mathbf{\Delta} \mathrm{AM})$ e em fêmeas $(\bullet \mathrm{AF})$ do rebanho Gir.

Figure 11 - Annual mean breeding values of bull's height at 378 days $(\mathbf{\Delta} A M)$ and heifer's height at 550 days $(\bullet \mathrm{AF})$ of the Gir herd.

se menores flutuações anuais na análise da mudança genética por MM, visto que a relação entre as gerações foi considerada na análise. Outro aspecto relevante é que, na estimativa de mudança genética por QM, a maioria dos desvios assumiram valores negativos, pois inicialmente as médias do $\mathrm{NeC}$ eram superiores às do Gir. Por outro lado, na estimativa de mudança genética por MM, ocorreram, para a maioria dos anos, valores positivos e crescentes no rebanho Gir, posto que, neste tipo de análise, a mudança genética é obtida como desvio do desempenho (valor genético em determinado ano) em relação ao valor genético dos animais-base. No rebanho $\mathrm{NeC}$, os valores genéticos para as características de seleção direta (P378 e P550) mantiveram-se próximos de zero, o que era esperado, pois neste rebanho a seleção é realizada com base em diferenciais de seleção próximos de zero. 
As estimativas de tendência genética para o rebanho Gir obtidas por meio de análises de QM, pelas diferenças entre os coeficientes de regressão linear nos rebanhos Gir e NeC, encontram-se na Tabela 3. Os valores encontrados foram 0,16 kg/ano para PN; $0,81 \mathrm{~kg} /$ ano para P210;3,02 g/dia/ano para GPRE; 2,88 kg/ano para P378; 11,2 g/dia/ano para G112; 2,80 $\mathrm{kg}$ /ano para P550; 0,003 m/ano para AM; e 0,004 m/ano para AF.

Na Tabela 3 constam também as estimativas de tendência genética obtidas por MM, as quais foram iguais a $0,03 \mathrm{~kg} / \mathrm{ano}$ (PN), 0,28 kg/ano (P210), 1,15 g/dia/ano (GPRE), 1,45 kg/ano (P378), 3,73 g/dia/ano (G112) e 1,07 kg/ano (P550), 0,003 m/ano (AM) e $0,002 \mathrm{~m} / \mathrm{ano}(\mathrm{AF})$ para o rebanho Gir.
Observando as estimativas obtidas pelas duas metodologias, verifica-se que as obtidas por QM foram geralmente superiores às de MM. Para que tendências genéticas anuais, estimadas por QM, sejam confiáveis, pelo menos três condições básicas devem ser atendidas: não deve ocorrer nenhuma seleção direcional na população controle originada da mesma população; a tendência fenotípica deve ser significativa somente em seu grau linear; e não deve ocorrer deriva genética aleatória. Como pelo menos as duas primeiras pressuposições não foram atendidas no rebanho estudado, pode-se inferir que isso torna a maior parte das estimativas por QM tendenciosa. Além disso,

Tabela 3 - Estimativas de tendências fenotípica, genética e ambiental para peso ao nascer (PN), peso à desmama corrigido para 210 dias (P210), ganho diário na fase pré-desmama (GPRE), peso de machos ao final da prova de ganho de peso (P378), ganho diário obtido na prova de ganho de peso (G112), peso de fêmeas corrigido para 550 dias (P550), altura aos 378 dias, em machos $(\mathrm{AM})$, e altura aos 550 dias, em fêmeas (AF) dos rebanhos Gir e Nelore Controle ( $\mathrm{NeC}$ )

Table 3 - Estimates of phenotypic, genetic and environmental trends for birth weight (PN), weaning weight corrected to 210 days (P210) daily weight gain in pre weaning phase (GPRE), male's performance test weight at 378 days (P378), daily weight gain during the performance test (G112), heifer's weight at 550 days (P550), bulls and heifers height at final weights (AF and AM) of the Gir and Nelore control (NeC) herds

\begin{tabular}{|c|c|c|c|c|}
\hline \multirow[t]{2}{*}{$\begin{array}{l}\text { Tendência } \\
\text { Trend }\end{array}$} & \multirow[t]{2}{*}{$\begin{array}{c}\text { Método } \\
\text { Method }\end{array}$} & \multirow[t]{2}{*}{$\begin{array}{c}\text { Característica } \\
\text { Trait }\end{array}$} & \multicolumn{2}{|c|}{$\begin{array}{c}\text { Rebanho } \\
\text { Herd }\end{array}$} \\
\hline & & & $\mathrm{NeC}$ & Gir \\
\hline Fenotípica (Phenotypic, kg/year) & $\mathrm{QM}(L S)$ & $\mathrm{PN}$ & $-0,08 \pm 0,02$ & $0,08 \pm 0,02$ \\
\hline Genética (Genetic, kg/year) & $\mathrm{QM}(L S)$ & $\mathrm{PN}$ & & $0,16 \pm 0,03$ \\
\hline Ambiental(Environmental, kg/year) & $\mathrm{QM}(L S)$ & $\mathrm{PN}$ & $-0,08 \pm 0,02$ & \\
\hline Genética (Genetic, kg/year) & $\mathrm{MM}(M M)$ & $\mathrm{PN}$ & & $0,03 \pm 0,01$ \\
\hline Fenotípica (Phenotypic, kg/year) & $\mathrm{QM}(L S)$ & P210 & $0,41 \pm 0,11$ & $1,22 \pm 0,11$ \\
\hline Genética (Genetic, kg/year) & $\mathrm{QM}(L S)$ & P2 10 & & $0,81 \pm 0,15$ \\
\hline Ambiental(Environmental, $\mathrm{kg} /$ year) & $\mathrm{QM}(L S)$ & P210 & $0,41 \pm 0,11$ & \\
\hline Genética (Genetic, kg/year) & $\mathrm{MM}(M M)$ & $\mathrm{P} 210$ & & $0,28 \pm 0,04$ \\
\hline Fenotípica (Phenotypic, g/day/year) & $\mathrm{QM}(L S)$ & GPRE & $2,42 \pm 0,50$ & $5,44 \pm 0,50$ \\
\hline Genética (Genetic, g/day/year) & $\mathrm{QM}(L S)$ & GPRE & & $3,02 \pm 0,71$ \\
\hline Ambiental(Environmental, g/day/year) & $\mathrm{QM}(L S)$ & GPRE & $2,42 \pm 0,50$ & \\
\hline Genética (Genetic, g/day/year) & $\mathrm{MM}(M M)$ & GPRE & & $1,15 \pm 0,17$ \\
\hline Fenotípica (Phenotypic, g/day/year) & $\mathrm{QM}(L S)$ & G112 & $1,0 \pm 0,20$ & $12,2 \pm 1,40$ \\
\hline Genética (Genetic, g/day/year) & $\mathrm{QM}(L S)$ & G112 & & $11,2 \pm 1,72$ \\
\hline Ambiental(Environmental, g/day/year) & $\mathrm{QM}(L S)$ & G112 & $1,0 \pm 0,20$ & \\
\hline Genética (Genetic, g/day/year) & $\mathrm{MM}(M M)$ & G112 & & $3,73 \pm 0,22$ \\
\hline Fenotípica (Phenotypic, kg/year) & $\mathrm{QM}(L S)$ & P378 & $0,45 \pm 0,21$ & $3,33 \pm 0,21$ \\
\hline Genética (Genetic, kgy/year) & $\mathrm{QM}(L S)$ & P378 & & $2,88 \pm 0,30$ \\
\hline Ambiental(Environmental, kg/year) & $\mathrm{QM}(L S)$ & P378 & $0,45 \pm 0,21$ & \\
\hline Genética (Genetic, kg/year) & $\mathrm{MM}(M M)$ & P378 & $-0,03 \pm 0,04$ & $1,45 \pm 0,08$ \\
\hline Fenotípica (Phenotypic, kg/year) & $\mathrm{QM}(L S)$ & P550 & $1,26 \pm 0,18$ & $4,06 \pm 0,18$ \\
\hline Genética (Genetic, kg/year) & $\mathrm{QM}(L S)$ & P550 & & $2,80 \pm 0,25$ \\
\hline Ambiental(Environmental, kg/year) & $\mathrm{QM}(L S)$ & P550 & $1,26 \pm 0,18$ & \\
\hline Genética (Genetic, kg/year) & $\mathrm{MM}(M M)$ & P550 & $0,05 \pm 0,05$ & $1,07 \pm 0,06$ \\
\hline Fenotípica (Phenotypic, m/year) & $\mathrm{QM}(L S)$ & $\mathrm{AM}$ & $-0,0033 \pm 0,0004$ & $-0,0006 \pm 0,0004$ \\
\hline Genética (Genetic, m/year) & $\mathrm{QM}(L S)$ & $\mathrm{AM}$ & & $0,003 \pm 0,0005$ \\
\hline Ambiental(Environmental, m/year) & $\mathrm{QM}(L S)$ & $\mathrm{AM}$ & $-0,0033 \pm 0,0004$ & \\
\hline Genética (Genetic, m/year) & $\mathrm{MM}(M M)$ & $\mathrm{AM}$ & & $0,003 \pm 0,0003$ \\
\hline Fenotípica (Phenotypic, m/year) & $\mathrm{QM}(L S)$ & $\mathrm{AF}$ & $-0,0011 \pm 0,0004$ & $0,0033 \pm 0,0004$ \\
\hline Genética (Genetic, m/year) & $\mathrm{QM}(L S)$ & $\mathrm{AF}$ & & $0,004 \pm 0,0005$ \\
\hline Ambiental (Environmental, m/year) & $\mathrm{QM}(L S)$ & $\mathrm{AF}$ & $-0,0011 \pm 0,0004$ & \\
\hline Genética (Genetic, m/year) & $\mathrm{MM}(M M)$ & $\mathrm{AF}$ & & $0,002 \pm 0,0003$ \\
\hline
\end{tabular}

${ }^{1}$ QM: quadrados mínimos, MM: modelo misto( LS: least squares; MM: mixed model). 
é possível perceber que as estimativas por MM forneceram menores erros-padrão.

Apesar da grande diferença verificada entre as estimativas de tendência genética pelos dois métodos, é válido afirmar que houve significativo progresso genético nas características de seleção direta (P378 e P550) no rebanho Gir. Quanto às demais características, os resultados obtidos indicaram que PN, P210, GPRE, G112, AM e AF apresentaram resposta correlacionada positiva à seleção para peso pós-desmama.

A estimativa de tendência genética anual obtida por MM para PN no rebanho Gir, equivalente a $30 \mathrm{~g} / \mathrm{ano}$, foi inferior a $42 \mathrm{~g} / \mathrm{ano}$ relatada por MacNeil et al. (1992), em bovinos da raça Hereford. Quanto ao P210, a estimativa de tendência genética obtida por MM foi bem inferior $(0,28 \mathrm{~kg} / \mathrm{ano})$ aos valores descritos na literatura, obtidos por quadrados mínimos para peso à desmama (Frahm et al., 1985a).

As respostas médias anuais para P378 e P550 verificadas neste estudo por modelo misto foram inferiores às obtidas em outros experimentos. Razook et al. (1998) obtiveram, pelo método QM, estimativas de tendência genética anual para P378 de 2,68 a 2,96 kg/ano em um rebanho Nelore e de 2,20 a 2,53 kg/ano em um rebanho Guzerá que fazem parte do projeto de seleção das raças zebuínas, estabelecido na EEZS. Para P550, esses mesmos autores encontraram valores de 2,63 a 2,81 kg/ano no rebanho Nelore e de 1,37 a 2,15 kg/ano no rebanho Guzerá. Baker et al. (1991) obtiveram respostas anuais para peso ao ano iguais a $2,49 \pm 0,24 \mathrm{~kg} /$ ano e a $2,16 \pm 0,31 \mathrm{~kg} /$ ano em duas linhas de Angus e Hereford, respectivamente, enquanto Koch et al. (1994) estimaram a resposta anual em 2,64 kg/ano em um rebanho Hereford. Mais recentemente, Mercadante et al. (2003) obtiveram estimativas por modelo animal de $1,70 \pm 0,22$ e $2,33 \pm 0,22 \mathrm{~kg} /$ ano em machos dos dois rebanhos Nelore de Sertãozinho, selecionados para peso aos 378 dias e 1,78 $\pm 0,20$ e 2,39 $\pm 0,20 \mathrm{~kg} /$ ano nas fêmeas selecionadas para peso aos 550 dias.

As estimativas de resposta anual para peso a um ano nos experimentos de seleção têm diferido não apenas pela metodologia utilizada, mas também pelas diferenças entre os intervalos de geração e as intensidades de seleção aplicadas, além das próprias diferenças populacionais.

As herdabilidades realizadas para P378 e P550, respectivamente de 0,69 e 0,64, foram bem superiores às estimativas encontradas na literatura para peso ao ano (Baker et al., 1991; Koch et al., 1994 ). Isso talvez tenha resultado do fato de a regressão linear, tendo como variável independente os diferenciais acumulados, não explicar totalmente a variação dos desvios obtidos do rebanho Gir em relação ao rebanho $\mathrm{NeC}$.

\section{Conclusões}

A seleção para peso pós-desmama no rebanho Gir com base na informação de desempenho individual promoveu respostas genéticas significativas para peso de machos ao final da prova de ganho de peso (P378) e para peso de fêmeas corrigido para 550 dias (P550), assim como as respostas correlacionadas para peso ao nascer, peso à desmama corrigido para 210 dias, ganho diário na fase pré-desmama, ganho diário obtido na prova de ganho de peso, altura aos 378 dias em machos e aos 550 dias em fêmeas.

Os critérios de seleção dos reprodutores foram atendidos em vista das significativas intensidades de seleção de touros e vacas no rebanho Gir.

As estimativas de tendência genética obtidas pelo método dos quadrados mínimos foram superiores e apresentaram maiores erros-padrão em relação às obtidas pela metodologia de modelos mistos sob modelo animal.

\section{Literatura Citada}

BAKER, R.L.; MORRIS, C.A.; JOHNSON, D.L. et al. Results of selection for yearling or 18-month weight in Angus and Hereford cattle. Livestock Production Science, v.29, p.277-296, 1991.

BOLDMAN, K.G.; KRIESE, L.A.; VAN VLECK, L.D. et al. A manual for use for MTDFREML: A set of programs to obtain estimates of variances and co variances. Lincoln: Department of Agriculture; Agricultural Research Service, 1995. 120p.

BRINKS, J.S.; CLARK, R.T.; RICE, F.J. Estimation of genetic trends in beef cattle. Journal of Animal Science, v.20, p.903, 1961.

CYRILLO, J.N.S.G.; RAZOOK, A.G.; FIGUEIREDO, L.A. et al. Efeitos da seleção para peso pós-desmame sobre medidas corporais e perímetro escrotal de machos Nelore de Sertãozinho (SP). Revista Brasileira de Zootecnia, v.29, p.403-412, 2000.

DICKERSON, G.E.; BLUNN, C.T.; CHAPMAN, A.B. et al. Evaluation of selection in developing inbred lines of swine, si. Missouri: Agricultural Experiment Station, 1954. 60p. (Research Bulletin, 551).

FALCONER, D.S.; MACKAY, T.F.C. Introduction to quantitative genetics. 4.ed. Essex: Longman, 1996. 464p.

FRAHM, R.R.; NICHOLS; C.G.; BUCHANAN, D.S. Selection for increased weaning or yearling weight in Hereford cattle. I. Measurement of selection applied. Journal of Animal Science, v.60, n.6, p.1373-1384, 1985a.

HARVEY, W.R. User's guide for LSMLMW and MIXMDL PC-2 version. Wooster: Ohio State Universiy, 1990. 91p.

KOCH, R.M.; CUNDIFF, L.V.; GREGORY, K.E. Cumulative selection and genetic change for weaning or yearling weight plus muscle score in Hereford cattle. Journal of Animal Science, v.72, p.864-885, 1994.

MacNEIL, M.D.; URICK, J.J.; NEWMAN, S. et al. Selection for postweaning growth in inbred Hereford cattle: the Fort Keogh, Montana Line 1 example. Journal of Animal Science, v.70, p.723-733, 1992.

MERCADANTE, M.E.Z.; PACKER, I.U.; RAZOOK, A.G. et al. Quadrados mínimos e modelo misto no acesso à estimativa da resposta direta à seleção em rebanhos experimentais de bovinos de corte. Revista Brasileira de Zootecnia, v.32, n.5, p.1123$1130,2003$.

MERCADANTE, M.E.Z.; PACKER, I.U.; RAZOOK, A.G. et al. Direct responses to selection for yearling weight on 
reproductive performance of Nelore cows. Journal of Animal Science, v.81, p.376-384, 2003.

NEWMAN, J.A.; RAHNEFELD, G.W.; FREDEEN, H.T. Selection intensity and response to selection for yearling weight in beef cattle. Canadian Journal of Animal Science, v.53, n.1, p.1-12, 1973.

PACKER, I.U.; RAZOOK, A.G.; TROVO, J.B.F. et al. Selection for yearling weight in Nelore and Guzerá breeds. Selection applied and response. In: WORLD CONGRESS ON GENETICS APPLIED TO LIVESTOCK PRODUCTION, 3., 1986, Lincoln. Proceedings... Lincoln: 1986. p.419-423.

POLASTRE, R.; TAMBURO, F.J.Z.; LAPERUTA FILHO, J. Manual para utilização do Sistema de Relacionamento Genético Animal (SIREGE). Botucatu: Universidade Estadual Paulista, 1992. 8p.

RAZOOK, A.G.; FIGUEIREDO, L.A.; BONILHA NETO, L.M. et al. Selection for yearling weight in Nelore and Guzerá breeds: selection applied and response in 15 years of progeny. In: WORLD CONGRESS OF GENETICS APPLIED TOLIVESTOCK PRODUCTION, 6., 1998, Armidale. Proceedings... Armidale: University of New England, 1998. v.23, p.133-136.
RAZOOK, A.G. Intensidade de seleção e mudanças genéticas em características de crescimento de rebanhos Nelore e Guzerá selecionados para peso pós-desmama. Estação Experimental de Zootecnia de Sertãozinho, 2002. 46p. (Relatório-Bolsa produtividade em pesquisa-CNPq).

STATISTICAL ANALYSES SYSTEM - SAS. SAS/STAT ${ }^{\text {TM. SAS }}$ user's guide for windows environment. version 6. 11.ed. Cary: 1999. 